\title{
Evolutionary Conservation Levels of Subunits of Histone-Modifying Protein Complexes in Fungi
}

\author{
Hiromi Nishida \\ Agricultural Bioinformatics Research Unit, Graduate School of Agricultural and Life Sciences, The University of Tokyo, 1-1-1 Yayoi, \\ Bunkyo-ku, Tokyo 113-8657, Japan \\ Correspondence should be addressed to Hiromi Nishida, hnishida@iu.a.u-tokyo.ac.jp
}

Received 20 August 2008; Revised 17 November 2008; Accepted 17 February 2009

Recommended by Graziano Pesole

Eukaryotes possess a variety of histone-modifying protein complexes. Generally, a histone-modifying protein complex consists of multiple subunits, that is, a catalytic subunit and the associated subunits. In this study, I analyzed 62 and 48 subunits of the histone-modifying protein complexes of Saccharomyces cerevisiae and Schizosaccharomyces pombe, respectively. The evolutionary conservation levels of the 110 subunits were measured. The measurements revealed that the conservation levels of the catalytic subunits are significantly higher than those of the associated subunits of the histone acetyltransferase and deacetylase complexes; however, the conservation level of the catalytic subunits is similar to that of the associated subunits of the histone methyltransferase complexes. Thus, in the fungal histone acetylation and deacetylation systems, the catalytic subunits of histone-modifying protein complexes are conserved and the associated subunits are evolutionary lineage-specific. In contrast, in the fungal histone methylation system, both the catalytic and the associated subunits are evolutionary lineage-specific.

Copyright () 2009 Hiromi Nishida. This is an open access article distributed under the Creative Commons Attribution License, which permits unrestricted use, distribution, and reproduction in any medium, provided the original work is properly cited.

\section{Introduction}

Chromatin is the most important structure for the maintenance of the eukaryotic genomic DNA. The eukaryotic genomic DNA is packaged with histone proteins to form nucleosomes (the fundamental repeating unit of chromatin). Chromatin structure depends on the modification of nucelosome core histones [1,2]. Generally the proteins that are evolutionarily conserved and distributed among a wide range of organisms play an important role in the biological processes [3]. Eukaryotes possess a variety of histone-modifying protein complexes $[4,5]$. Some subunits of histone-modifying protein complexes have been evolutionarily conserved among eukaryotes, while some subunits are evolutionary lineage-specific. For example, the histonemodifying protein Clr4 methylates histone $\mathrm{H} 3$ at lysine 9, which plays a major role in RNA-mediated heterochromatin formation in the fission yeast $S$. pombe [5]. However, the budding yeast Sacchromyces cerevisiae lacks Clr4 homologue [6]. There has been considerable progress in the studies on fungal histone modifications in S. cerevisiae and S. pombe. Most gene functions in other fungi have been annotated based on the structural similarity of their genes with the genes of the abovementioned 2 yeasts whose functions have been studied well (inferred from the results of biological experiments). In this study, I used the subunits of histonemodifying protein complexes extracted from Saccharomyces and Schizosaccharomyces. The purpose of this study is to show the evolutionary conservation levels of the subunits of fungal histone-modifying protein complexes.

\section{Materials and Methods}

The sequences of coding for subunits of histone-modifying protein complexes in Saccharomyces and Schizosaccharomyces were extracted from 2 major genome databases, Saccharomyces Genome Database (http://www.yeastgenome .org/), and S. pombe GeneDB (http://www.genedb.org/ genedb/pombe/) at the Wellcome Trust Sanger Institute. In order to identify proteins homologous to the extracted Saccharomyces and Schizosaccharomyces proteins, a BLASTP search was performed for 9 complete fungal genomes (6 ascomycetes species, namely, Aspergillus 
TABLE 1: Evolutionary conservation levels of Saccharomyces proteins related to histone modifications.

\begin{tabular}{|c|c|c|c|c|c|c|c|c|c|c|}
\hline Protein & Function & & Scor & base & d on & valu & in $B$ & ASTP & & \\
\hline & & $\mathrm{kla}$ & yli & spo & $\mathrm{ncr}$ & $\mathrm{afm}$ & cne & uma & $\mathrm{ecu}$ & Tot: \\
\hline ELP3 & HAT catalytic & 5 & 5 & 5 & 5 & 5 & 5 & 5 & 5 & 40 \\
\hline RPD3 & HDAC catalytic & 5 & 5 & 4 & 5 & 5 & 5 & 5 & 4 & 38 \\
\hline HOS2 & HDAC catalytic & 5 & 5 & 4 & 4 & 4 & 4 & 4 & 4 & 34 \\
\hline TRA1 & HAT associated & 5 & 5 & 5 & 5 & 5 & 5 & 2 & 2 & 34 \\
\hline ESA1 & HAT catalytic & 5 & 5 & 4 & 4 & 4 & 4 & 4 & 3 & 33 \\
\hline GCN5 & HAT catalytic & 5 & 5 & 4 & 4 & 4 & 4 & 4 & 3 & 33 \\
\hline HDA1 & HDAC catalytic & 5 & 5 & 5 & 4 & 3 & 4 & 4 & 2 & 32 \\
\hline TAF1 & HAT catalytic & 5 & 5 & 4 & 3 & 4 & 3 & 4 & 3 & 31 \\
\hline SIN3 & HDAC associated & 5 & 4 & 3 & 5 & 4 & 3 & 4 & 2 & 30 \\
\hline SET2 & HMT catalytic & 5 & 3 & 4 & 2 & 4 & 3 & 3 & 2 & 26 \\
\hline HST1 & HDAC catalytic & 5 & 3 & 3 & 3 & 3 & 3 & 3 & 2 & 25 \\
\hline NTO1 & HAT catalytic & 5 & 3 & 3 & 3 & 3 & 3 & 3 & 2 & 25 \\
\hline SAS3 & HAT catalytic & 4 & 3 & 3 & 3 & 3 & 3 & 3 & 3 & 25 \\
\hline HAT2 & HAT associated & 4 & 3 & 3 & 3 & 3 & 3 & 3 & 2 & 24 \\
\hline HOS3 & HDAC catalytic & 5 & 4 & 2 & 3 & 3 & 2 & 3 & 2 & 24 \\
\hline RPH1 & HDMT catalytic & 4 & 4 & 2 & 3 & 3 & 3 & 3 & 2 & 24 \\
\hline SIR2 & HDAC catalytic & 4 & 3 & 3 & 3 & 3 & 3 & 3 & 2 & 24 \\
\hline JHD2 & HDMT catalytic & 5 & 3 & 3 & 3 & 3 & 3 & 3 & 0 & 23 \\
\hline HST2 & HDAC catalytic & 3 & 2 & 3 & 3 & 3 & 3 & 3 & 2 & 22 \\
\hline SET1 & HMT catalytic & 5 & 3 & 2 & 3 & 3 & 2 & 2 & 2 & 22 \\
\hline SWD1 & HMT associated & 4 & 3 & 3 & 3 & 2 & 2 & 2 & 2 & 21 \\
\hline EAF1 & HAT associated & 5 & 3 & 2 & 2 & 2 & 2 & 2 & 2 & 20 \\
\hline EPL1 & HAT associated & 4 & 3 & 2 & 2 & 3 & 2 & 2 & 2 & 20 \\
\hline SWD2 & HMT associated & 4 & 3 & 2 & 3 & 2 & 2 & 2 & 2 & 20 \\
\hline HAT1 & HAT catalytic & 4 & 3 & 3 & 2 & 2 & 3 & 2 & 0 & 19 \\
\hline SAS2 & HAT catalytic & 4 & 3 & 2 & 2 & 2 & 2 & 2 & 2 & 19 \\
\hline SET3 & HDAC associated & 4 & 3 & 2 & 2 & 2 & 2 & 2 & 2 & 19 \\
\hline SIF2 & HDAC associated & 4 & 3 & 2 & 2 & 2 & 2 & 2 & 2 & 19 \\
\hline GIS1 & HDMT catalytic & 3 & 3 & 2 & 2 & 2 & 2 & 2 & 2 & 18 \\
\hline HDA3 & HDAC catalytic & 5 & 3 & 1 & 2 & 2 & 1 & 2 & 2 & 18 \\
\hline HOS1 & HDAC catalytic & 4 & 2 & 2 & 2 & 2 & 2 & 2 & 2 & 18 \\
\hline JHD1 & HDMT catalytic & 4 & 3 & 2 & 2 & 3 & 2 & 2 & 0 & 18 \\
\hline SPT10 & HAT catalytic & 4 & 3 & 2 & 2 & 3 & 2 & 2 & 0 & 18 \\
\hline SPP1 & HMT associated & 3 & 2 & 2 & 2 & 2 & 2 & 2 & 2 & 17 \\
\hline SWD3 & HMT associated & 3 & 2 & 2 & 2 & 2 & 2 & 2 & 2 & 17 \\
\hline YAF9 & HAT associated & 3 & 2 & 2 & 2 & 2 & 2 & 2 & 2 & 17 \\
\hline YNG2 & HAT associated & 3 & 2 & 2 & 2 & 2 & 2 & 2 & 2 & 17 \\
\hline BRE2 & HMT associated & 4 & 2 & 2 & 2 & 2 & 2 & 2 & 0 & 16 \\
\hline NAT4 & HAT catalytic & 2 & 2 & 2 & 2 & 2 & 2 & 2 & 2 & 16 \\
\hline PHO23 & HDAC catalytic & 2 & 2 & 2 & 2 & 2 & 2 & 2 & 2 & 16 \\
\hline RTT109 & HAT catalytic & 4 & 2 & 2 & 2 & 2 & 2 & 2 & 0 & 16 \\
\hline SAS5 & HAT catalytic & 2 & 2 & 2 & 2 & 2 & 2 & 2 & 2 & 16 \\
\hline YNG1 & HAT associated & 2 & 2 & 2 & 2 & 2 & 2 & 2 & 2 & 16 \\
\hline DOT1 & HMT catalytic & 4 & 3 & 0 & 2 & 2 & 2 & 2 & 0 & 15 \\
\hline RCO1 & HDAC associated & 3 & 2 & 2 & 2 & 2 & 2 & 2 & 0 & 15 \\
\hline SGF29 & HAT associated & 3 & 2 & 2 & 2 & 2 & 2 & 2 & 0 & 15 \\
\hline SWC4 & HAT associated & 3 & 2 & 2 & 2 & 2 & 2 & 2 & 0 & 15 \\
\hline HPA2 & HAT catalytic & 2 & 2 & 2 & 2 & 2 & 2 & 2 & 0 & 14 \\
\hline RXT3 & HDAC associated & 2 & 2 & 2 & 2 & 2 & 2 & 2 & 0 & 14 \\
\hline SGF73 & HAT associated & 2 & 2 & 2 & 2 & 2 & 2 & 2 & 0 & 14 \\
\hline EAF7 & HAT associated & 2 & 2 & 2 & 2 & 0 & 2 & 2 & 0 & 12 \\
\hline RXT2 & HDAC associated & 3 & 2 & 2 & 2 & 2 & 0 & 0 & 0 & 11 \\
\hline SDC1 & HMT associated & 2 & 2 & 2 & 2 & 2 & 0 & 0 & 1 & 11 \\
\hline SGF11 & HAT associated & 2 & 2 & 2 & 0 & 2 & 1 & 2 & 0 & 11 \\
\hline AHC1 & HAT associated & 3 & 2 & 0 & 2 & 2 & 0 & 0 & 0 & 9 \\
\hline EAF6 & HAT catalytic & 2 & 2 & 1 & 2 & 2 & 0 & 0 & 0 & 9 \\
\hline HDA2 & HDAC catalytic & 3 & 2 & 1 & 1 & 1 & 1 & 0 & 0 & 9 \\
\hline HIF1 & HAT associated & 2 & 0 & 1 & 2 & 1 & 2 & 0 & 0 & 8 \\
\hline SAP30 & HDAC catalytic & 2 & 2 & 1 & 1 & 2 & 0 & 0 & 0 & 8 \\
\hline SAS4 & HAT catalytic & 2 & 2 & 1 & 2 & 1 & 0 & 0 & 0 & 8 \\
\hline SHG1 & HMT associated & 2 & 2 & 2 & 0 & 1 & 0 & 0 & 0 & 7 \\
\hline RFM1 & HDAC associated & 2 & 0 & 0 & 0 & 1 & 0 & 0 & 0 & 3 \\
\hline
\end{tabular}

HAT, histone acetyltransferase; HDAC, histone deacetylase;

HDMT, histone demethylase; HMT, histone methyltransferase

afm, Aspergillus fumigatus; ecu, Encephalitozoon cuniculi; cne, Cryptococcus neoformans; kla, Kluyveromyces lactis; ncr, Neurospora crassa; spo, Schizosaccharomyces pombe; uma, Ustilago maydis; yli, Yarrowia lipolytica. 


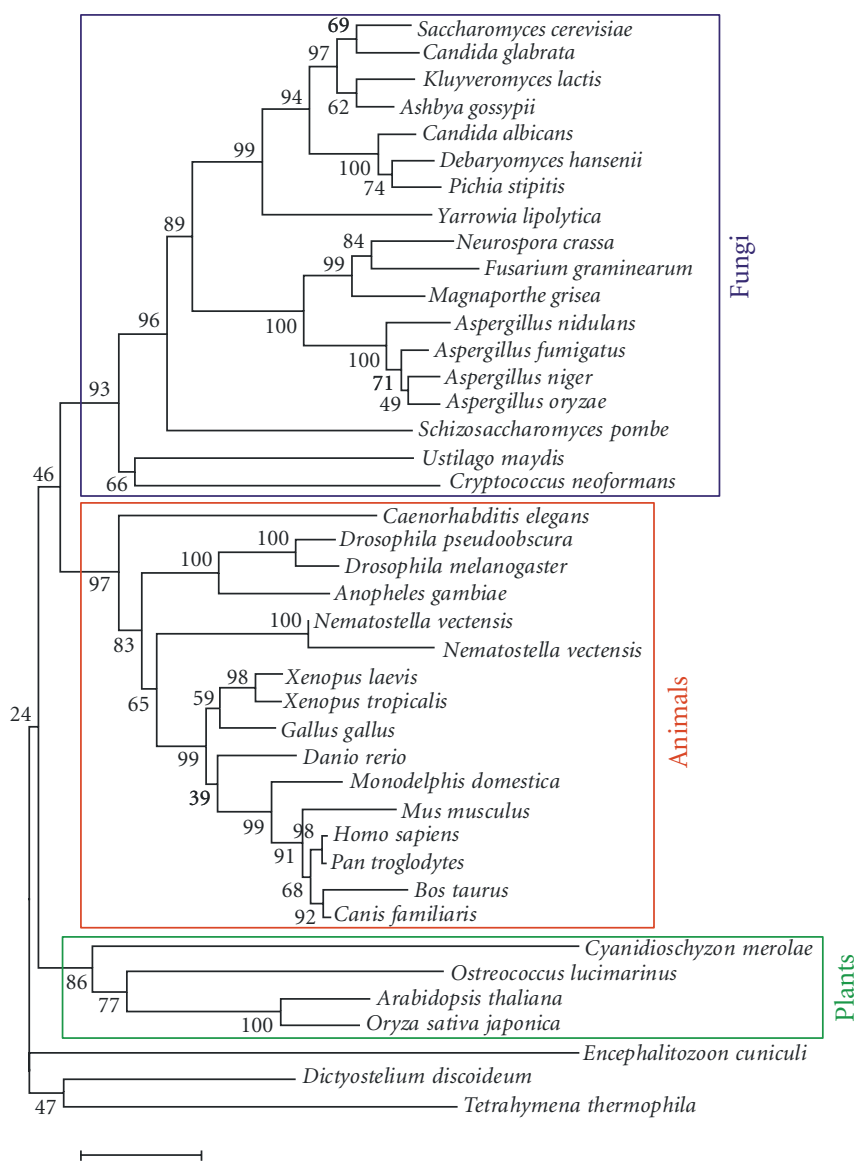

(a)

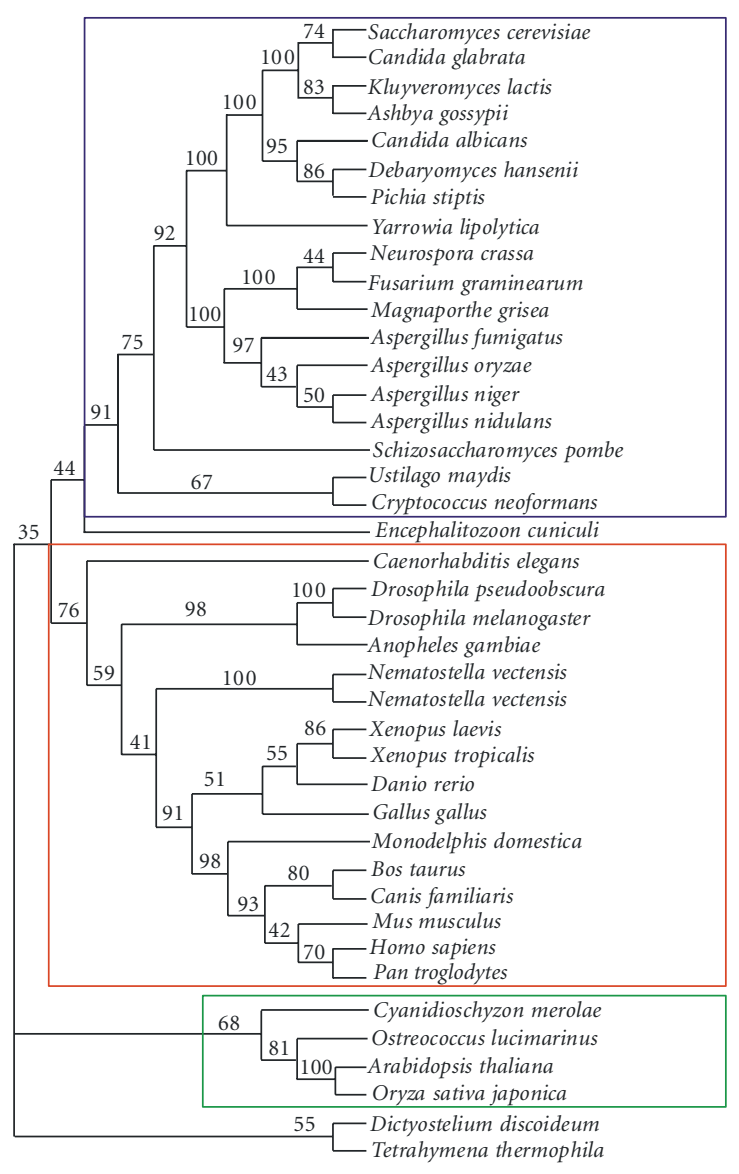

(b)

FIGURE 1: Phylogenetic relationships among the histone acetyltransferase catalytic subunit ELP3 and its homologues. A total of 496 amino acid sites were considered from multiple alignments with all the gap sites deleted. (a) Neighbor-joining tree was generated with 1000 bootstrap replicates using the MEGA software [8]. The number at each node represents the percentage obtained in the bootstrap analysis. The bar indicates a 5\% difference in the evolutionary distance. (b) Maximum likelihood tree was generated with 100 bootstrap replicates using the PHYLIP software [9]. The number at each node represents the percentage obtained in the bootstrap analysis. The JTT model was used as the model of amino acid substitution. Number of times to jumble in the PROML program was 2.

fumigatus, Kluyveromyces lactis, Neurospora crassa, S. cerevisiae, S. pombe, and Yarrowia lipolytica; 2 basidiomycetes species, namely, Cryptococcus neoformans, Ustilago maydis, and 1 microsporidium, namely, Encephalitozoon cuniculi) in the Kyoto Encyclopedia of Genes and Genomes (KEGG) database [7]. Based on the $E$ values of the BLASTP search results, I classified 6 evolutionary conservation levels and scored them as follows: score 0 , not detected; score 1, $E$ value $>10^{0}$; score $2,10^{-50}<E$ value $\leq 10^{0}$; score $3,10^{-100}<E$ value $\leq 10^{-50} ;$ score $4,10^{-150}<E$ value $\leq 10^{-100} ;$ score $5, E$ value $\leq 10^{-150}$. Based on the total of their scores, the subunits were ranked.

The Molecular Evolutionary Genetics Analysis (MEGA) software [8] was used to generate a neighbor-joining tree with 1000 bootstrap replicates from multiple alignments with all the gap sites deleted. A total of 496 amino acid sites were considered. PHYLIP software [9] was used to generate a maximum likelihood tree with 100 bootstrap replicates. The JTT model was used as the model of amino acid substitution. Number of times to jumble in the PROML program was 2.

\section{Results and Discussion}

From the 2-yeast genome databases, I extracted 62 and 48 subunits of histone-modifying protein complexes of S. cerevisiae and S. pombe, respectively. Among the 110 subunits, 34 Saccharomyces and 24 Schizosaccharomyces proteins were catalytic subunits; the others were associated complex subunits. The evolutionary conservation levels of the 62 Saccharomyces and 48 Schizosaccharomyces proteins are shown in Tables 1 and 2, respectively.

The histone acetyltransferase (HAT) catalytic subunit ELP3 was found to be the most conserved among the 62 Saccharomyces and 48 Schizosaccharomyces subunits. The main acetylation sites of ELP3 are lysine-14 of histone H3 and lysine- 8 of histone H4 [10]. In addition, ELP3 is an integral subunit of elongating RNA polymerase II holoenzyme in S. cerevisiae, which is involved in transcriptionassociated chromatin modification and remodeling $[11,12]$. The deletion of ELP3 gene in yeast confers slow growth adaptation, slow gene activation, and temperature sensitivity 
TABLE 2: Evolutionary conservation levels of Schizosaccharomyces proteins related to histone modifications.

\begin{tabular}{|c|c|}
\hline Protein & Function \\
\hline ELP3 & HAT catalytic \\
\hline CLR6 & HDAC catalytic \\
\hline HOS2 & HDAC catalytic \\
\hline TRA1 & HAT associated \\
\hline CLR3 & HDAC catalytic \\
\hline GCN5 & HAT catalytic \\
\hline MST1 & HAT catalytic \\
\hline SET2 & HMT catalytic \\
\hline PST1 & HDAC associated \\
\hline MST2 & HAT catalytic \\
\hline PRW1 & HDAC associated \\
\hline JMJ2 & HDMT catalytic \\
\hline SET1 & HMT catalytic \\
\hline HST2 & HDAC catalytic \\
\hline NTO1 & HAT associated \\
\hline PST2 & HDAC associated \\
\hline SIR2 & HDAC catalytic \\
\hline SWD1 & HMT associated \\
\hline HST4 & HDAC catalytic \\
\hline SPAC25H1.06 & HAT associated \\
\hline SWD2 & HMT associated \\
\hline SWD3 & HMT associated \\
\hline CLR4 & HMT catalytic \\
\hline EPL1 & HAT associated \\
\hline RTT109 & HAT catalytic \\
\hline SET3 & HMT catalytic \\
\hline SPAC17G8.07 & HAT catalytic \\
\hline SPCC1235.09 & HDAC associated \\
\hline CTI6 & HDAC associated \\
\hline HAT1 & HAT catalytic \\
\hline SPAC22E12.19 & HDAC associated \\
\hline ALP13 & HDAC associated \\
\hline CPH1 & HDAC associated \\
\hline $\mathrm{CPH} 2$ & HDAC associated \\
\hline LSD1 & HDMT catalytic \\
\hline LSD2 & HDMT catalytic \\
\hline SET5 & HMT catalytic \\
\hline SET6 & HMT catalytic \\
\hline SET9 & HMT catalytic \\
\hline SPCC1795.08c & HAT associated \\
\hline SPP1 & HMT associated \\
\hline SWC4 & HAT associated \\
\hline SET7 & HMT catalytic \\
\hline SPAC6F6.09 & HAT associated \\
\hline EAF7 & HAT associated \\
\hline SPBC428.06c & HDAC associated \\
\hline SDS3 & HDAC associated \\
\hline SPCC126.13c & HDAC associated \\
\hline
\end{tabular}

\begin{tabular}{ccccccccc}
\multicolumn{7}{c}{ Score based on $E$ value in BLASTP } \\
sce & kla & yli & ncr & afm & cne & uma & ecu & Total \\
5 & 5 & 5 & 5 & 5 & 5 & 5 & 5 & 40 \\
5 & 5 & 5 & 5 & 5 & 5 & 5 & 4 & 39 \\
4 & 4 & 5 & 4 & 4 & 5 & 4 & 4 & 34 \\
5 & 5 & 5 & 5 & 5 & 5 & 2 & 2 & 34 \\
4 & 5 & 5 & 4 & 4 & 4 & 4 & 2 & 32 \\
4 & 4 & 4 & 4 & 4 & 4 & 4 & 3 & 31 \\
4 & 4 & 4 & 4 & 4 & 4 & 4 & 3 & 31 \\
4 & 4 & 4 & 4 & 4 & 4 & 3 & 2 & 29 \\
3 & 3 & 4 & 4 & 4 & 3 & 3 & 2 & 26 \\
3 & 3 & 3 & 3 & 4 & 3 & 3 & 3 & 25 \\
3 & 3 & 3 & 3 & 3 & 3 & 3 & 3 & 24 \\
3 & 3 & 3 & 3 & 3 & 3 & 3 & 2 & 23 \\
3 & 3 & 3 & 3 & 3 & 3 & 3 & 2 & 23 \\
3 & 3 & 2 & 3 & 3 & 3 & 3 & 2 & 22 \\
3 & 3 & 2 & 3 & 3 & 3 & 3 & 2 & 22 \\
3 & 3 & 3 & 2 & 3 & 3 & 3 & 2 & 22 \\
3 & 3 & 2 & 3 & 3 & 3 & 3 & 2 & 22 \\
3 & 3 & 3 & 3 & 3 & 3 & 2 & 2 & 22 \\
3 & 3 & 3 & 3 & 3 & 2 & 2 & 2 & 21 \\
2 & 2 & 2 & 3 & 3 & 3 & 3 & 2 & 20 \\
2 & 2 & 3 & 2 & 3 & 2 & 2 & 2 & 18 \\
2 & 2 & 3 & 2 & 3 & 2 & 2 & 2 & 18 \\
2 & 2 & 2 & 2 & 2 & 2 & 2 & 2 & 16 \\
2 & 2 & 2 & 2 & 2 & 2 & 2 & 2 & 16 \\
2 & 2 & 2 & 2 & 2 & 2 & 2 & 2 & 16 \\
2 & 2 & 2 & 2 & 2 & 2 & 2 & 2 & 16 \\
2 & 2 & 2 & 2 & 2 & 2 & 2 & 2 & 16 \\
2 & 2 & 2 & 2 & 2 & 2 & 2 & 2 & 16 \\
2 & 2 & 2 & 2 & 2 & 2 & 2 & 1 & 15 \\
3 & 2 & 2 & 2 & 2 & 2 & 2 & 0 & 15 \\
2 & 2 & 2 & 2 & 2 & 2 & 2 & 1 & 15 \\
2 & 2 & 2 & 2 & 2 & 2 & 2 & 0 & 14 \\
2 & 2 & 2 & 2 & 2 & 2 & 2 & 0 & 14 \\
2 & 2 & 2 & 2 & 2 & 2 & 2 & 0 & 14 \\
2 & 2 & 2 & 2 & 2 & 2 & 2 & 0 & 14 \\
1 & 2 & 2 & 2 & 2 & 2 & 2 & 1 & 14 \\
2 & 2 & 2 & 2 & 2 & 2 & 2 & 0 & 14 \\
2 & 2 & 2 & 2 & 2 & 2 & 2 & 0 & 14 \\
2 & 2 & 2 & 2 & 2 & 2 & 2 & 0 & 14 \\
2 & 2 & 2 & 2 & 2 & 2 & 2 & 0 & 14 \\
2 & 2 & 2 & 2 & 2 & 2 & 2 & 0 & 14 \\
2 & 2 & 2 & 2 & 2 & 2 & 2 & 0 & 14 \\
2 & 2 & 2 & 2 & 1 & 2 & 2 & 0 & 13 \\
2 & 2 & 2 & 2 & 2 & 1 & 2 & 0 & 13 \\
1 & 1 & 2 & 2 & 0 & 2 & 0 & 1 & 11 \\
0 & 2 & 2 & 2 & 2 & 0 & 0 & 0 & 8 \\
2 & 1 & 1 & 0 & 1 & 1 & 1 & 0 & 7 \\
\hline & & & & & & & &
\end{tabular}

HAT, histone acetyltransferase; HDAC, histone deacetylase; HDMT, histone demethylase; HMT, histone methyltransferase.

afm, Aspergillus fumigatus; ecu, Encephalitozoon cuniculi; cne, Cryptococcus neoformans; kla, Kluyveromyces lactis; ncr, Neurospora crassa; sce, Saccharomyces cerevisiae; uma, Ustilago maydis; yli, Yarrowia lipolytica

[11]. The ELP3 protein's function may be so important for fungi (eukaryotes) that it is the most conserved.

The phylogenetic tree based on ELP3 and its homologues show that these proteins are present across eukaryotes (Figures $1(\mathrm{a})$ and $1(\mathrm{~b}))$. The phylogenetic relationships among fungal ELP3 and its homologues are consistent with the fungal classification [13]. Interestingly, the microsporidium E. cuniculi is not included in the fungal lineage in the neighbor-joining tree (Figure 1(a)), but it is included in the maximum likelihood tree with $44 \%$ bootstrap support (Figure 1(b)).

There are some lineage-specific subunits of histonemodifying protein complexes. For example, homologues of Dot1, the histone methyltransferase (HMT) catalytic subunit of Saccharomyces are present in K. lactis and Y. lipolytica-2 ascomycetous yeasts (Table 1). Dot1 methylates the histone $\mathrm{H} 3$ at lysine-79, which is related to gene silencing in $S$. cerevisiae [14]. This modification system is also found in 


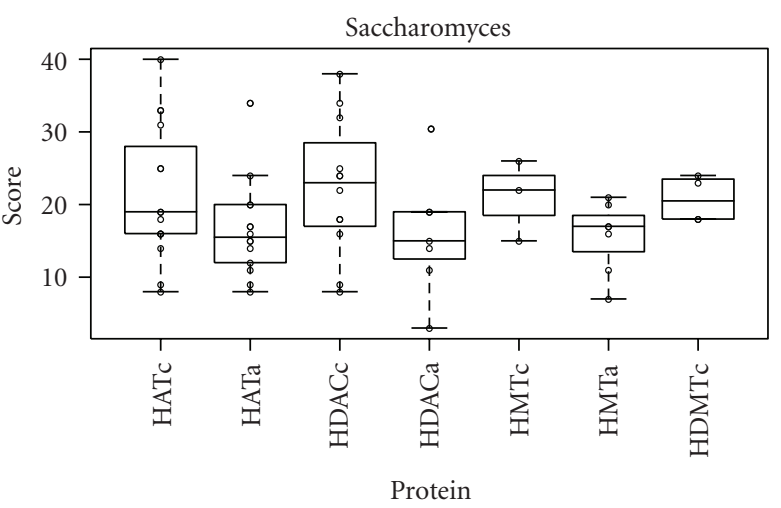

(a)

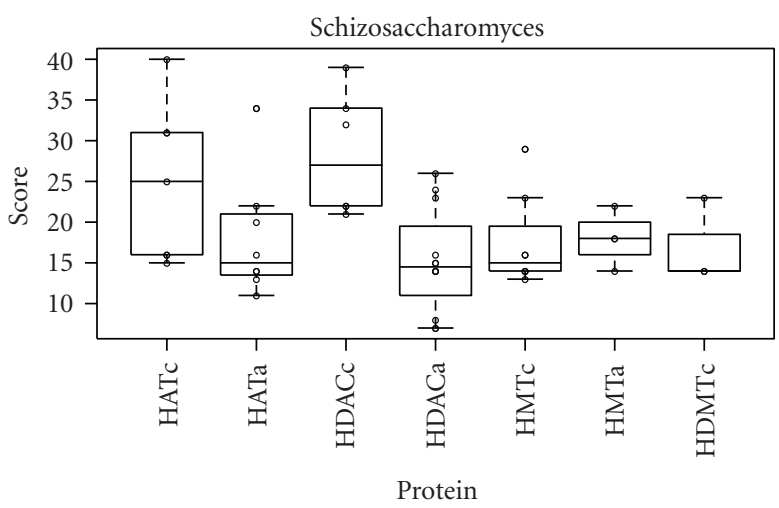

(b)

FIGURE 2: Boxplots of evolutionary conservation levels of subunits of histone-modifying protein complexes. Based on the $E$ values of the BLASTP search results, I classified 6 evolutionary conservation levels and scored them as follows: score 0 , not detected; score $1, E$ value $>10^{0}$; score $2,10^{-50}<E$ value $\leq 10^{0}$; score $3,10^{-100}<E$ value $\leq 10^{-50}$; score $4,10^{-150}<E$ value $\leq 10^{-100}$; score $5, E$ value $\leq 10^{-150}$. Each circle indicates the total of the scores of each protein. Top and bottom boxplots are based on the Saccharomyces and Schizosaccharomyces proteins, respectively. Boxes are composed of medians with first and third quartiles from the scores of the subunits. HATc, histone acetyltransferase (HAT) catalytic subunits; HATa, HAT-associated subunits; HDACc, histone deacetylase (HDAC) catalytic subunits; HDACa, HDACassociated subunits; HMTc, histone methyltransferase (HMT) catalytic subunits; HMTa, HMT-associated subunits; and HDMTc, histone demethylase catalytic subunits.

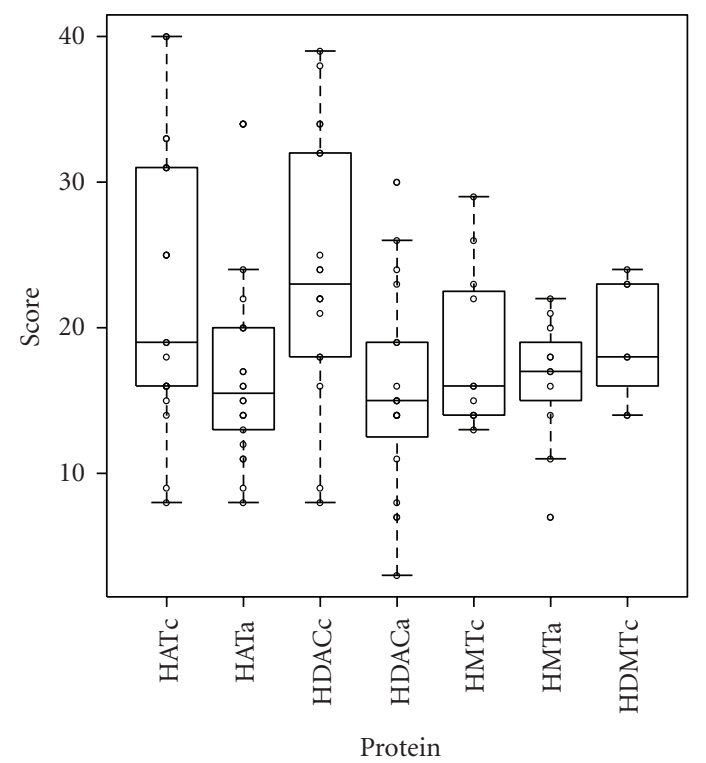

FIGURE 3: Boxplots of combined evolutionary conservation levels of Saccharomyces and Schizosaccharomyces subunits of histonemodifying protein complexes. Based on the $E$ values of the BLASTP search results, I classified 6 evolutionary conservation levels and scored them as follows: score 0 , not detected; score $1, E$ value $>10^{0}$; score $2,10^{-50}<E$ value $\leq 10^{0}$; score $3,10^{-100}<E$ value $\leq 10^{-50}$; score $4,10^{-150}<E$ value $\leq 10^{-100}$; score $5, E$ value $\leq 10^{-150}$. Each circle indicates the total of the scores of each protein. Boxes are composed of medians with first and third quartiles from the scores of the subunits. HATc, histone acetyltransferase (HAT) catalytic subunits; HATa, HAT-associated subunits; HDACc, histone deacetylase (HDAC) catalytic subunits; HDACa, HDACassociated subunits; HMTc, histone methyltransferase (HMT) catalytic subunits; HMTa, HMT-associated subunits; HDMTc, histone demethylase catalytic subunits. mammals [14]. However, S. pombe has no homologue of Dot1 (Table 1).

The evolutionary conservation levels of the HAT and histone deacetylase (HDAC) catalytic subunits are higher than those of the associated subunits, respectively, in Saccharomyces and Schizosaccharomyces (Figure 2). However, the conservation levels of the HMT catalytic subunits are similar to those of the associated subunits, especially in Schizosaccharomyces (Figure 2). In order to elucidate the difference in evolutionary conservation levels, I analyzed the combined data of the conservation scores of the HAT catalytic and the associated subunits, HDAC catalytic and the associated subunits, HMT catalytic and the associated subunits, and the histone demethylase (HDMT) catalytic subunits of Saccharomyces and Schizosaccharomyces. Distributions of the conservation levels of the combined data are shown in Figure 3 . The $P$ values obtained by the Wilcoxon rank-sum test for the difference between the conservation levels of the HAT catalytic and the associated subunits, HDAC catalytic and the associated subunits, and HMT catalytic and the associated subunits were $.043(<.05), .0027(<.05)$, and .90 (>.05), respectively. Thus, the null hypothesis (conservation levels of catalytic and the associated subunits are equal) was rejected in the case of the histone acetylation and deacetylation systems, but not for the histone methylation system.

The results of this study show that histone acetylase and deacetylase catalytic subunits are more conserved than other subunits (Figures 2 and 3). In fact, out of the 10 most abundant proteins of Saccharomyces and Schizosaccharomyces, 9 were histone acetylation or deacetylation related proteins, that is, 4 HAT catalytic, 3 HDAC catalytic, 1 HAT associated, 1 HDAC associated, and 1 HMT catalytic subunits 
(Tables 1 and 2). In fungal histone acetylation and deacetylation, the catalytic subunits of protein complexes are conserved and the associated subunits are evolutionary lineagespecific. However, in fungal histone methylation, both the catalytic and the associated subunits are evolutionary lineage-specific. Although the histone modification systems work cooperatively, these results strongly suggest that the evolution of the fungal histone acetylation/deacetylation system was different from that of the histone methylation system.

\section{Acknowledgment}

This study was supported by a Grant from the Institute for Fermentation, Osaka.

\section{References}

[1] B. D. Strahl and C. D. Allis, "The language of covalent histone modifications," Nature, vol. 403, no. 6765, pp. 41-45, 2000.

[2] T. Jenuwein and C. D. Allis, "Translating the histone code," Science, vol. 293, no. 5532, pp. 1074-1080, 2001.

[3] R. L. Tatusov, M. Y. Galperin, D. A. Natale, and E. V. Koonin, "The COG database: a tool for genome-scale analysis of protein functions and evolution," Nucleic Acids Research, vol. 28 , no. 1, pp. 33-36, 2000.

[4] K. K. Lee and J. L. Workman, "Histone acetyltransferase complexes: one size doesn't fit all," Nature Reviews Molecular Cell Biology, vol. 8, no. 4, pp. 284-295, 2007.

[5] C. Martin and Y. Zhang, "The diverse functions of histone lysine methylation," Nature Reviews Molecular Cell Biology, vol. 6, no. 11, pp. 838-849, 2005.

[6] C. B. Millar and M. Grunstein, "Genome-wide patterns of histone modifications in yeast," Nature Reviews Molecular Cell Biology, vol. 7, no. 9, pp. 657-666, 2006.

[7] M. Kanehisa, S. Goto, M. Hattori, et al., "From genomics to chemical genomics: new developments in KEGG," Nucleic Acids Research, vol. 34, database issue, pp. D354-D357, 2006.

[8] K. Tamura, J. Dudley, M. Nei, and S. Kumar, "MEGA4: Molecular Evolutionary Genetics Analysis (MEGA) software version 4.0," Molecular Biology and Evolution, vol. 24, no. 8, pp. 1596-1599, 2007.

[9] J. Felsenstein, PHYLIP (Phylogeny Inference Package), version 3.67, Department of Genome Sciences and Department of Biology, University of Washington, Seattle, Wash, USA.

[10] G. S. Winkler, A. Kristjuhan, H. Erdjument-Bromage, P. Tempst, and J. Q. Svejstrup, "Elongator is a histone H3 and $\mathrm{H} 4$ acetyltransferase important for normal histone acetylation levels in vivo," Proceedings of the National Academy of Sciences of the United States of America, vol. 99, no. 6, pp. 3517-3522, 2002.

[11] B. Ø. Wittschieben, G. Otero, T. de Bizemont, et al., "A novel histone acetyltransferase is an integral subunit of elongating RNA polymerase II holoenzyme," Molecular Cell, vol. 4, no. 1, pp. 123-128, 1999.

[12] B. Ø. Wittschieben, J. Fellows, W. Du, D. J. Stillman, and J. Q. Svejstrup, "Overlapping roles for the histone acetyltransferase activities of SAGA and Elongator in vivo," EMBO Journal, vol. 19, no. 12, pp. 3060-3068, 2000.

[13] D. S. Hibbett, M. Binder, J. F. Bischoff, et al., "A higher-level phylogenetic classification of the Fungi," Mycological Research, vol. 111, no. 5, pp. 509-547, 2007.
[14] Q. Feng, H. Wang, H. H. Ng, et al., "Methylation of H3-lysine 79 is mediated by a new family of HMTases without a SET domain," Current Biology, vol. 12, no. 12, pp. 1052-1058, 2002. 

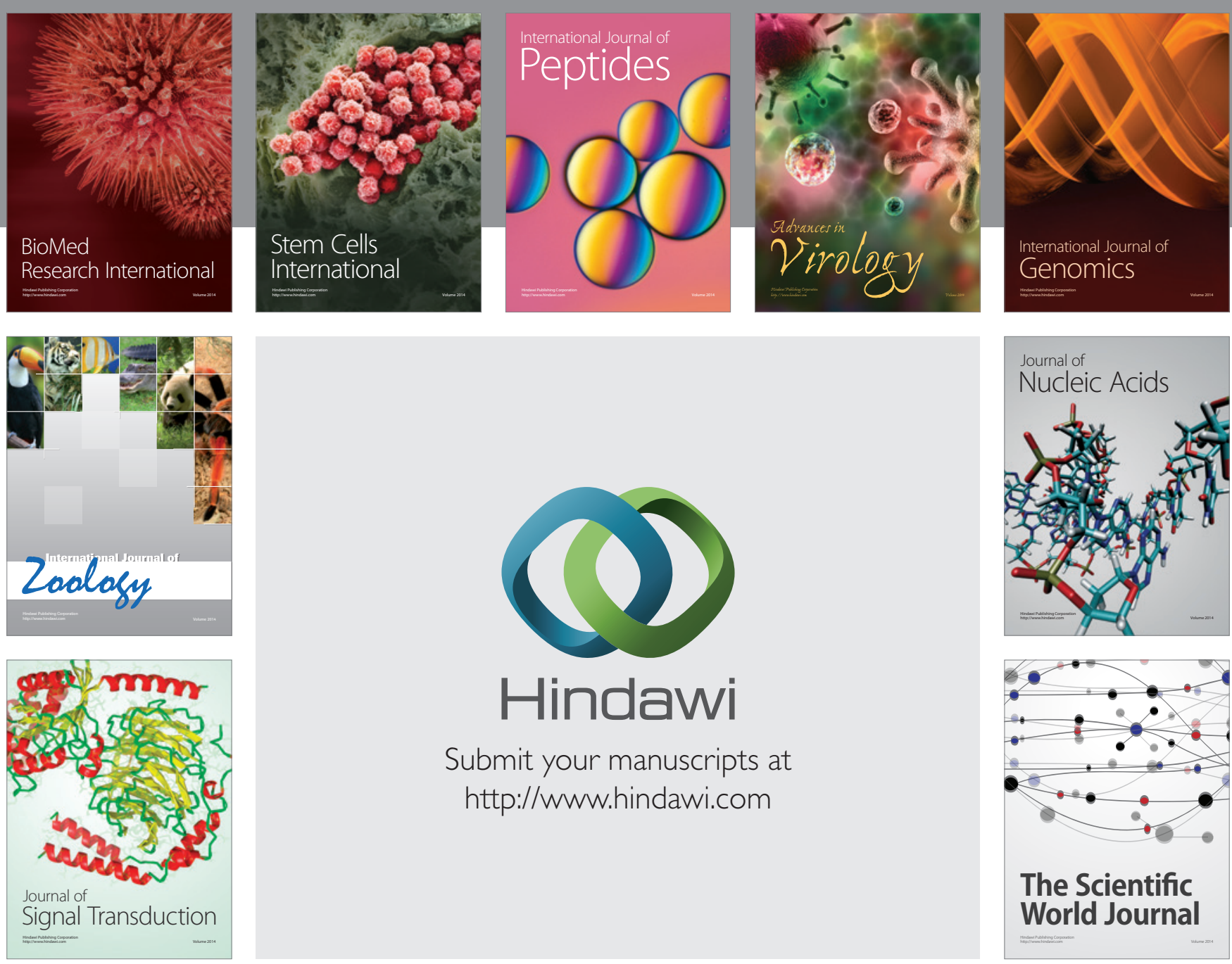

Submit your manuscripts at

http://www.hindawi.com
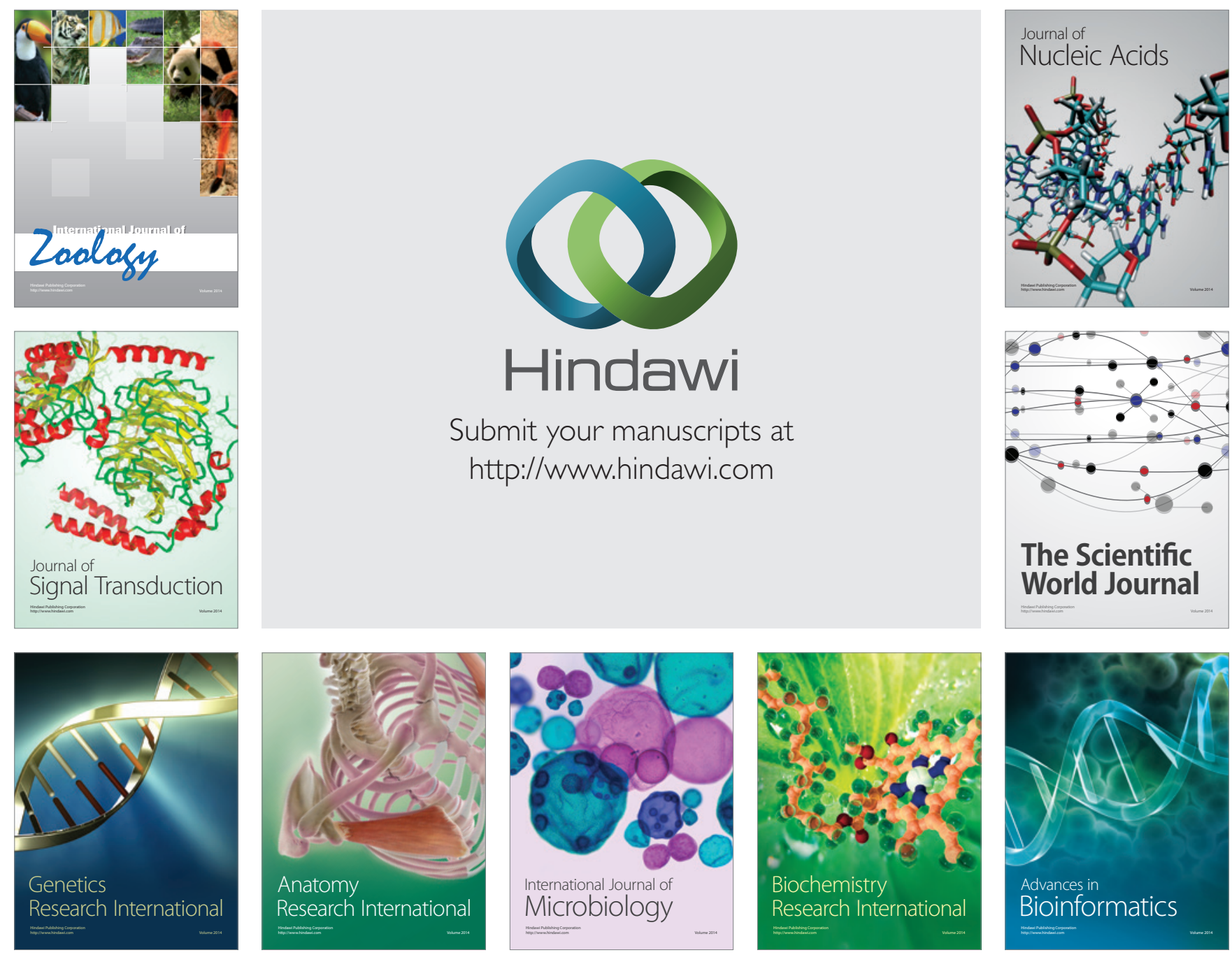

The Scientific World Journal
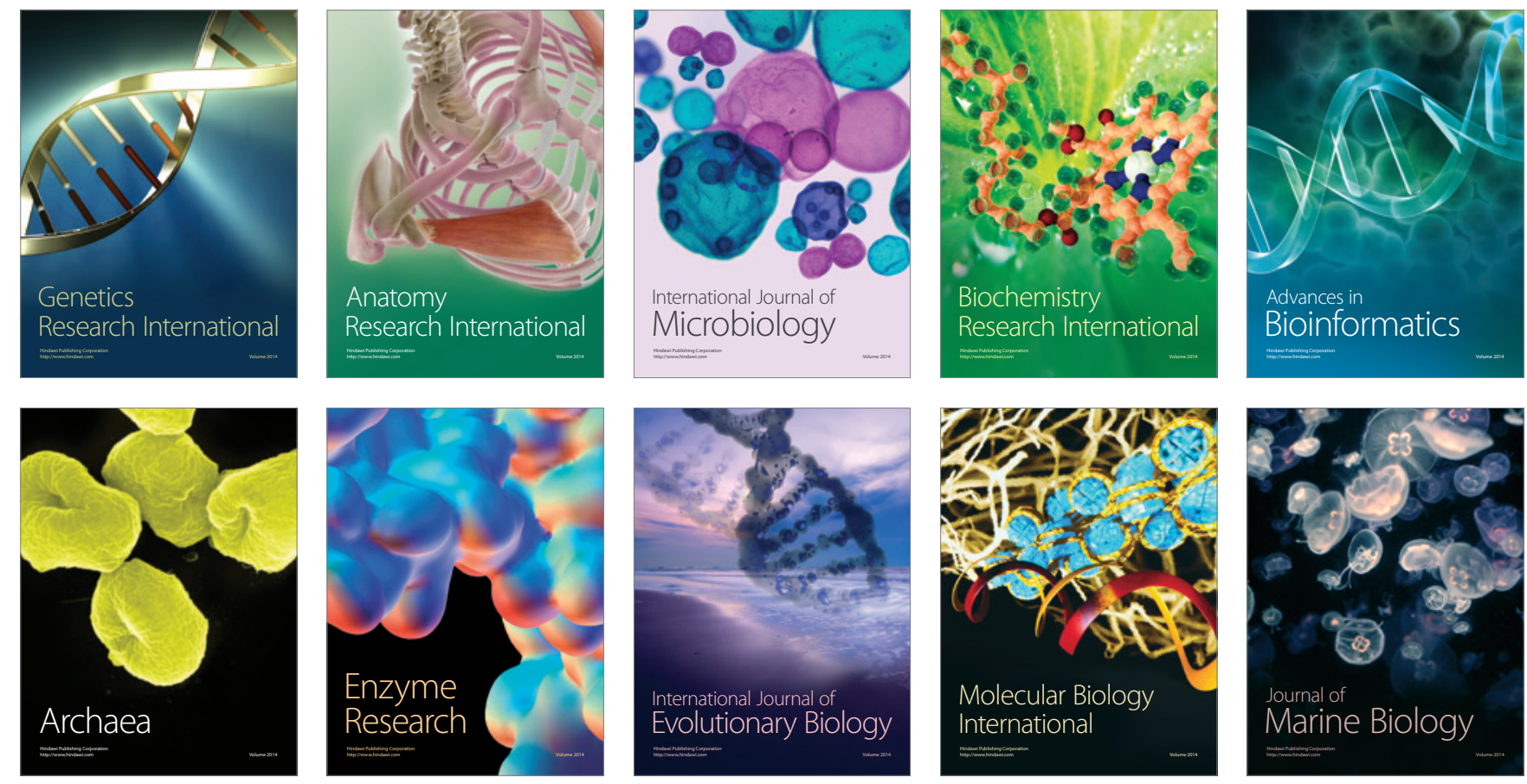